\title{
A CASE REPORT ON DECOMPENSATED CIRRHOSIS OF LIVER WITH PREGNANCY
}

\author{
MOHAMMAD MAHABUBUL ALAM ${ }^{1}$, FAIZ AHMAD KHONDAKAR ${ }^{2}$, SYED ABUL FOEZ ${ }^{1}$, MAMUN AL-MAHTAB ${ }^{1}$, \\ SALIMUR RAHMAN ${ }^{1}$
}

\begin{abstract}
Pregnancy in women with advanced liver disease is rare. Cirrhosis results in metabolic and hormonal derangements that lead to anovulation and amenorrhea ${ }^{1}$. In this paper we described the case of a successful pregnancy in a young woman with advanced cirrhosis due to hepatitis B virus infection.
\end{abstract}

Key words: Hepatitis $B$, liver cirrhosis, viable pregnancy

\section{Introduction}

Pregnancy is uncommon in women with advanced cirrhosis and is associated with an increased risk of complications such as bleeding from esophageal varices, liver failure and hepato-renal syndrome ${ }^{1-4}$. Esophageal variceal bleeding has been reported in $18 \%$ to $32 \%$ of pregnant women with cirrhosis and in up to $50 \%$ of those with known portal hypertension ${ }^{4,5}$. Among those with preexisting varices, up to $78 \%$ will have gastrointestinal bleeding during pregnancy, with a mortality rate of $18 \%$ to $50 \%{ }^{1}$. Maternal deaths have been reported in advanced cirrhosis mainly due to variceal bleeding 4 . Maternal mortality is likely to improve with better management of variceal hemorrhage and liver failure. Up to $24 \%$ of pregnant patients with cirrhosis will also experience hepatic decompensation, which can lead to rapid clinical deterioration. When fulminant hepatic failure occurs, the only treatment available is liver transplantation.

Spontaneous abortion and increased risk of premature childbirth or stillbirth have been reported in $15 \%-20 \%$ of pregnancies in women with cirrhosis ${ }^{5}$. We are reporting a case of a successful pregnancy outcome of a woman with decompensated cirrhosis, caused by hepatitis $B$ virus.

\section{Case Report}

The patient, a 28 year old married female got admitted into Department of Obstetrics \& Gynaecology, Bangabandhu Sheikh Mujib Medical University (BSMMU) with the complaints of amenorrhoea for last 9 months and gradual abdominal distention and swelling of both lower limbs for last 5 months.
According to the statement of the patient, she was relatively well at 5 months back with 4 months of viable pregnancy. She noticed sudden onset of vomiting of blood and passage of tarry colored stool for 3 days. With these complaints she got admitted in a hospital and was treated conservatively with blood transfusion. From there she was transferred to BSMMU and was diagnosed as a case of HBVrelated cirrhosis of liver with 4 months viable pregnancy. Emergency endoscopy of upper gastrointestinal tract (UGIT) and oesophageal variceal ligation (EVL) was done. She was discharged with diuretics and B-blocker. She continued regular follow up under an Obstetrician. After 2 months, diuretics were stopped and swelling of her both lower limbs and abdomen became worse. The patient subsequently got admitted into BSMMU again for better management.

She had no history of jaundice and gave birth of a healthy child 5 years back. On examination, she was

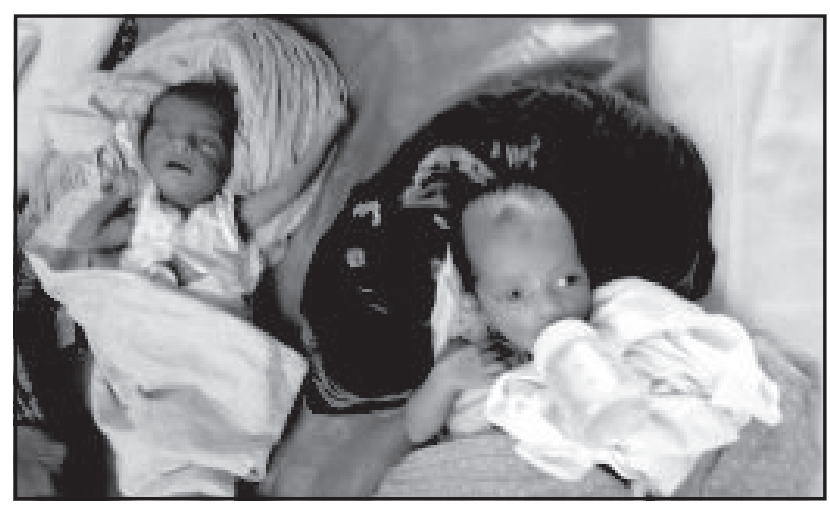

Fig.-1

1. Dept of Hepatology, Bangabandhu Sheikh Mujib Medical University, Dhaka

2. Noakhali Medical College \& Hospital, Noakhali.

Bangladesh J Medicine 2011; 22 : 60-62 
conscious and well-cooperative. She was mildly anaemic, but not ecteric. Pulse rate was $78 / \mathrm{min}$, regular and blood pressure was $100 / 70 \mathrm{~mm}$ of $\mathrm{Hg}$. Abdomen was distended, umbilicus everted with striae graviderum in lower abdomen. Ascites was evidenced by shifting dullness. No organomegaly was noted. Other systemic examination revealed normal study.

Investigations revealed hemoglobin of $11.2 \mathrm{gm} / \mathrm{dl}$ with total leukocyte count of $12000 / \mathrm{mm}^{3}(85 \%$ polymorphs and $10 \%$ lymphocytes), platelets $1,40,000 / \mathrm{mm}^{3}$, total billirubin 34.0 microm $/ 1$, serum glutamic oxaloacetic transaminase (ALT) $42 \mathrm{U} / \mathrm{L}$ (UNL $37 \mathrm{U} / \mathrm{L})$, serum glutamic pyruvic transaminase $24 \mathrm{U} /$ L (AST) (UNL $65 \mathrm{U} / \mathrm{L}$ ) and alkaline phosphatase 397 U/L (UNL136), serum albumin $2.1 \mathrm{gm} / \mathrm{dl}$ and prothrombin time 16.0 seconds (control 11.8 seconds), activated partial thromboplastin time 42.0 seconds (control 28 seconds), fibrin degradation product 10.0 ug/dl (normal <5 ug/dl), D-dimer $1.5 \mathrm{ug} /$ dl (normal $<0.5)$. She had normal renal function and electrolytes.

Abdominal and pelvic ultrasound (USG) revealed a shrunken liver, mild spleenomegaly, moderate ascities and 19 weeks viable twin pregnancy with breech presentation and marked ascites. She was positive for HBsAg by ELISA. However, HBeAg by ELISA and HBV DNA by PCR were not detected in her serum. Repeat endoscopy of UGIT showed grade II-III esophageal varices and portal hypertensive gastropathy (PHG), but no gastric varices. EVL (second session) of esophageal varices was performed.

She was given propranolol $20 \mathrm{mg}$ twice daily and a diuretic (combination of frusemide $40 \mathrm{mg}+$ spironolactone $100 \mathrm{mg}$ ) $1 / 2$ tab daily. She underwent therapeutic paracentesis of massive ascities at $28^{\text {th }}$ week of gestation.

She eventually gave birth of twin babies. Normal vaginal delivery was accomplished. Both babies are in good in health and weight. But mother developed profuse post-partum bleeding, which was treated with FFP and inj. Vitamin K. Two units of whole fresh human blood was also transfused. Bleeding stopped after 3 days.

\section{Discussion}

Infertility is common even in mild forms of chronic liver diseases. Advanced cirrhosis increases the risk of maternal and fetal morbidity and mortality ${ }^{1,2}$. In such cases, the stage of the liver disease is the most important determinant of the outcome of the pregnancy $^{1,2,6,7}$.

In contrast to cirrhosis due to autoimmune etiology or alcoholic liver disease, the outcome of pregnancies in women with other types of chronic liver disease, especially of viral etiology, is poorly reported and therefore uncertain ${ }^{8}$.

Maternal death rate in women with cirrhosis is reported to be $10.3 \%$ to $18 \%$ with massive gastrointestinal bleed as the commonest cause of death $^{1,3}$ and liver failure as the next most frequent cause $^{2}$. In a review of 117 pregnancies, term pregnancy without maternal complications was achieved in 50\% of cases, while deterioration in liver function was observed in 44.4\%. Haematemesis occurred on 24 occasions and was responsible for maternal deaths in $4 \%$ of patients ${ }^{9}$.

Portal hypertension due to cirrhosis compounds the physiological increase in circulating blood volume, elevation in portal pressure and added pressure from the gravid uterus on the inferior vena cava and can result in massive bleeding. It is most common during the 2nd trimester with $20-27 \%$ chance of bleeding from esophageal varices, which is amplified to 62$78 \%$ if there are large size varices ${ }^{1-4,7}$. Therefore, it is mandatory to assess such patients for portal hypertension, which can be done by indirect evidence, such as the presence of esophageal varices, abdominal collateral veins, hypersplenism and ascites. Endoscopic variceal band ligation or sclerotherapy and beta-blockers are the therapeutic options for such patients $^{2,3}$.

There is an increased rate of spontaneous abortion, premature birth and perinatal deaths in pregnant woman with advanced cirrhosis. Poor fetal prognosis is usually explained by poor condition of the mother in decompensated patients. Though, infants born alive generally remain well2,3.

In a controlled setting vaginal delivery is usually safe and early forceps delivery or vacuum extraction should be considered to prevent any rise in portal pressure due to prolonged straining during labor ${ }^{2,3}$. Women with cirrhosis generally tolerate laparotomy poorly; therefore the option for caesarean section should be availed with care and caution. Our patient had an uncomplicated vaginal delivery with moderate amount of postpartum bleeding which was controlled with konakion, FFP and blood transfusion. She did not have further hepatic decompensation, sepsis or any other complication.

\section{Conclusion}

Data related to the optimal management and outcome of pregnancy in women with decompensated cirrhosis secondary to viral etiology is limited. Whether to advise a pregnancy to a woman with decompensated cirrhosis is a difficult question to answer. However, 
careful overall assessment of the severity of the liver disease as well as of the patient's psychological status and desire for children should lead logically to a resolution of these issues on a case by case basis. With careful monitoring and advanced management, successful pregnancy with a good outcome is a good possibility. The excellent outcome of the pregnancy in our patient is encouraging and supports this opinion.

\section{References}

1. Tiribelli C, Rigato I. Liver cirrhosis and pregnancy. Ann Hepatol. 2006;5:201.

2. Aggarwal N, Sawnhey H, Suril V, Vasishta K, Jha M, Dhiman RK. Pregnancy and cirrhosis of the liver. Aust N Z J Obstet Gynaecol. 1999;39:503-6.

3. Cerqui AJ, Haran M, Brodribb R. Implications of liver cirrhosis in pregnancy. Aust $\mathrm{N} \mathrm{Z} \mathrm{J} \mathrm{Obstet}$ Gynaecol. 1998; 38: 93-5.
4. Zeeman GG, Moise KJ., Jr Prophylactic banding of severe esophageal varices associated with liver cirrhosis in pregnancy. Obstet Gynecol. 1999; 94:842. doi: 10.1016/S0029-7844(99)00438-X.

5. Lee WM. Pregnancy in patients with chronic liver disease. Gastroenterol Clin North Am. 1992; 21:889-903.

6. Whelton MJ, Sherlock S. Pregnancy in patients with hepatic cirrhosis. Management and outcome. Lancet. 1968;2:995-9. doi: 10.1016/S0140-6736(68)91294-4.

7. Garcia-Tsao G. Portal hypertension. Curr Opin Gastroenterol. 2006;22:254-62. doi: 10.1097/ 01.mog.0000218962.93806.9a.

8. Lee MG, Hanchard B, Donaldson EK, Charles C, Hall JS. Pregnancy in chronic active hepatitis with cirrhosis. Journal of tropical med \& hygiene. 1987;90:245-48.

9. Cheng YS. Pregnancy in liver cirrhosis and/or portal hypertension. Am J Obs Gynecol. 1977;128:812-22. 